\title{
SISTEMAS INTELIGENTES Y COLABORATIVOS DE ADMINISTRACIÓN GLOBAL: CASO DE ESTUDIO; ESTABLECIMIENTO GASTRONÓMICO
}

\author{
INTELLIGENT AND COLLABORATIVE SYSTEM OF \\ GLOBAL ADMINISTRATION FOR A GASTRONOMIC \\ ESTABLISHMENT
}

GABRIEL VINICIO MOREANO SÁNCHEZ VÍCTOR HUGO BENÍTEZ BRAVO² WILMER ALBARRACÍN ${ }^{3}$ ÁlVARO GABRIEL BENÍTEZ BRAVO

Recibido: 21 de diciembre de 2017 Aceptado: 31 de enero de 2018

\footnotetext{
${ }^{1}$ Escuela Politécnica del Chimborazo, Riobamba, Ecuador (gabriel.moreano@espoch.edu.ec).

${ }^{2}$ Universidad de las Fuerzas Armadas, ESPE, Quito, Ecuador, (vhbenitez@espe.edu.ec).

${ }^{3}$ Escuela de Ingeniería Universidad Tecnológica Israel, Quito, Ecuador, (walbarracin@uisrael.edu.ec).

${ }^{4}$ Universidad Central del Ecuador, Quito, Ecuador, (agbenitez@uce.edu.ec).
} 
illl|l 336 


\section{SISTEMA INTELIGENTE Y COLABORATIVO DE ADMINISTRACIÓN GLOBAL PARA UN ESTABLECIMIENTO GASTRONÓMICO}

\section{INTELLIGENT AND COLLABORATIVE SYSTEM OF GLOBAL ADMINISTRATION FOR A GASTRONOMIC ESTABLISHMENT}

Gabriel Moreano Sánchez, Víctor Hugo Benítez Bravo, Wilmer Albarracín, Álvaro Benítez Bravo

Palabras Clave: Metodología tradicional, metodología ágil, gestión de proyectos, plataforma tecnológica, SCRUM.

Keywords: waterfall methodology, Agile methodology, project management, technological platform, SCRUM.

\section{RESUMEN}

El rápido desarrollo tecnológico que abraza a nuestra era se genera para cubrir necesidades que tienen los seres humanos en su vida cotidiana, es decir, la tecnología avanza en pro de facilitar la sobrevivencia del ser humano y ayudarlo en sus labores diarias. Entre las prioridades del ser humano están la familia, el hogar y el trabajo, siendo este último el campo donde el ser humano quiere demostrar su valía y eficiencia y por tanto es el ámbito donde la tecnología trata de aportar más herramientas.

El trabajo de un restaurante puede ser desde el más sencillo del mundo hasta el más complicado y exigente según la demanda existente; en este documento se presenta el plan de ges- 
tión para la creación de una plataforma tecnológica que brinde herramientas de gestión y atención al cliente eficientes y eficaces capaces de mejorar y facilitar las tareas de atención, supervisión, control y administración en un establecimiento gastronómico.

Para la implementación de dicha plataforma se propone combinar metodologías de gestión tradicionales y ágiles, obteniendo como resultado un plan de proyecto donde se estiman alcances, costes y duración de las tareas de desa- rrollo, se definen los planes de gestión de calidad y de mitigación de riesgos y se integra una etapa de gestión de desarrollo mediante la metodología SCRUM que, a su vez, utilizará las estimaciones previas como métricas de evolución para identificar posibles retrasos e inconvenientes con el trabajo realizado. Con la combinación de metodologías, se ha intentado generar una solución eficiente para la administración del desarrollo de la plataforma tecnológica.

\section{ABSTRACT}

The technological development that embraces our era is generated to meet the needs of human beings in their daily lives, that is, technology advances to facilitate the survival of human beings and help them in their daily tasks. Among the priorities of the human being are the family, home and work, the latter being the field where the human being wants to demonstrate their worth and efficiency and therefore is the area where technology tries to provide more tools.

Work in a restaurant may be the simplest in the world or the most complicated and demanding according to the existing demand; This document presents the management plan for the creation of a technological platform that provides efficient and effective management and customer service tools capable of improving and facilitating the tasks of attention, supervision, control and administration in a gastronomic establishment.

For the implementation of this platform it is proposed to combine the traditional and agile management methodologies, obtaining as a result a project plan where the scope, costs and duration of the development tasks are estimated, the quality management and mitigation plans are defined of risks and a stage of development management is integrated through the SCRUM methodology, which in turn will use the previous estimates as evolution metrics to identify 
possible delays and inconveniences with the work done. With the combination of methodologies an attempt has been made to generate an efficient solution for the administration of the development of the technological platform.

\section{INTRODUCCIÓN}

Según el Instituto Ecuatoriano de Estadística y Censos (INEC)', en el año 2015, el $29 \%$ de todas las empresas a nivel nacional se dedicaron a la actividad hotelera y gastronómica y además han generado un $21 \%$ de empleos de entre toda la población activa del Ecuador; según la misma información se conoce que los ecuatorianos destinan el $5 \%$ de su presupuesto a consumición en locales alimenticios, llegando a una cifra global de 48.27 millones de dólares mensuales, esta información refleja que el negocio gastronómico representa un fuerte componente económico de la sociedad ecuatoriana.
La calidad de servicio de un establecimiento gastronómico representa un punto crítico en el éxito del negocio ya que generalmente los clientes valoran el sabor de las comidas, la atención recibida, el precio y la comodidad del lugar.

Por los antecedentes mencionados, se requiere el apoyo de las tecnologías de la información y la comunicación a fin de asegurar la calidad y rapidez en ciertos servicios como la toma de pedidos y el registro de productos, de esta manera se podrá aumentar la productividad de un establecimiento gastronómico.

\section{PLANTEAMIENTO DEL TRABAJO}

El problema real en este tipo de establecimientos es que, en ocasiones, la demanda que se tiene supera las capacidades de servicio, lo cual provoca un cuello de botella entre atención y servicio que desemboca en fallos de la atención como entrega equivocada de pedidos, molestia de los clientes por saturación de personas, mala atención por estrés del personal, pérdidas económicas por pedidos no entregados o facturas no emitidas, mala contabilidad, mal inventario de productos y posible pérdida de clientela, esto puede ser solventado con

\footnotetext{
${ }^{1}$ http://www.ecuadorencifras.gob.ec/hoteles-restaurantes-y-servicios/, Junio de 2017.
} 
la contratación de más personal, aumentando los costes de funcionamiento o mediante la aplicación de tecnologías que ayuden a liberar de ciertas tareas al personal del establecimiento gastronómico.

Otro de los problemas que motiva la realización del presente trabajo es los costos y las capacidades que pueden presentar sistemas de administración actuales, algunos de ellos se centran solo en tareas de facturación y contabilidad desestimando las labores de inventario y servicio al cliente. Otro tipo de aplicaciones cubren tareas de facturación, contabilidad y servicios como pedidos, pero mantienen la deficiencia de no ahondar en tareas de inventario, domicilios y gestión de cocina. A pesar de no satisfacer todas las áreas de administración que requeriría un establecimiento gastronómico, estos sistemas pueden llegar a ser muy costosos, en un promedio se estima que un sistema de facturación y servicio de pedidos puede llegar a costar $\$ 1500$ en Ecuador sin contabilizar el hardware necesario.
Restaurantes con la capacidad de llegar a ser franquicias nacionales e incluso internacionales suelen limitarse en su afán de crecimiento empresarial por las dificultades en la administración. La problemática surge desde la extrema dependencia del personal humano para realizar las tareas de servicio, razón por la cual una microempresa que desee expandir su negocio necesitará obligatoriamente contratar más personal asumiendo todos los riesgos que esto implica como falta de experiencia, posible contratación de personal conflictivo, falta de valores y lealtad hacia la empresa, incremento de costes de funcionamiento y aumento de la nómina de personal sobre quien se debe gestionar las actividades.

Además, al ser la nómina de trabajadores compacta, el administrador tendrá mayor facilidad en la asignación de tareas y en el control de las mismas pero se saturará de tareas al poco personal del que se dispone, provocando la caída tanto en la calidad como en la rapidez del servicio.

\section{FORMULACIÓN DEL PROBLEMA}

¿Se puede desarrollar un sistema inteligente que facilite las tareas de administración, gestión y atención al cliente de un restaurante? 


\section{JUSTIFICACIÓN}

Se propone la creación de un sistema inteligente y colaborativo que optimice el tiempo y la capacidad laboral de los trabajadores de un restaurante, que puede ser de nuevo emprendimiento o ya existente, mediante una asistencia tecnológica evitando tareas repetitivas de baja y media prioridad como traspaso de pedidos, generación de facturas, actualización automática de inventario y control del flujo de caja.

Si bien la idea principal es deslindar a los trabajadores de ciertas tareas, lo que se busca en esencia es mejorar la calidad de servicio hacia el cliente, y mediante esta mejora conseguir metas a corto plazo como aumentar las ventas diarias, atraer a más clientes, empoderarse del mercado local y mejorar el sistema administrativo del establecimiento y también lograr metas a largo plazo como motivar la expansión a varias sucursales locales y nacionales y manejar una base de datos general que mantenga los registros de compras y ventas de todas las sucursales.

Lograr las metas propuestas tanto a corto como a largo plazo generará un incremento en las ganancias de los socios o dueños de los restaurantes.

\section{OBJETIVOS}

\section{Objetivo General}

Desarrollar el plan de proyecto que permita implementar un sistema inteligente y colaborativo que dé soporte tecnológico en establecimientos gastronómicos, a fin de mejorar en tiempo y calidad, las labores de administración y gestión, así como las tareas de servicio al cliente.

\section{Objetivos Específicos}

- Realizar una propuesta viable para el desarrollo de una plataforma inteligente que facilite el trabajo en todas las áreas de un restaurante, administración, cocina y servicio al cliente.

- Seleccionar adecuadamente una metodología de trabajo para el seguimiento y control del proyecto. Identificar a los posibles interesados del proyecto, sean estos: entes tecnológicos de desarrollo, instituciones de financiamiento públicos o privados, entes de regulación de innovación y potenciales clientes y usuarios del sistema.

- Realizar un levantamiento de requerimientos en forma de historias de 
usuarios siguiendo las recomendaciones de la metodología SCRUM.

- Desarrollar el plan de trabajo que permita llegar a la implementación efectiva del sistema inteligente, esto es, el cronograma de actividades y el presupuesto general estimado para el proyecto.

- Realizar la descomposición de trabajo que permita un mayor control en la evolución del proyecto, definir funciones, roles y asignar responsabilidades a todos los miembros del equipo de trabajo.

- Definir el plan de aseguramiento de la calidad para los procesos y los resultados del proyecto, establecer las métricas adecuadas de calidad y los puntos de control.

- Identificar los riesgos potenciales que puedan afectar la marcha exitosa del proyecto y generar un plan de contingencias.

- Establecer el plan de comunicaciones que facilite la interacción entre los miembros del equipo de trabajo y de los representantes del mismo con entes externos.

\section{Objetivos del Proyecto}

- Dimensionar e instalar un servidor web que proporcione el servicio y soporte a todo el sistema colaborativo.

- Crear una base de datos universal de acceso inalámbrico.

- Diseñar y desarrollar una aplicación para Tablet que genere los pedidos digitales realizados por el personal de servicio de un restaurante.

- Diseñar y desarrollar una aplicación móvil para el uso de clientes.

- Diseñar y desarrollar un sistema de inventario que tenga comunicación directa con la base de datos universal.

- Implementar un sistema de visualización de pedidos para el personal de cocina.

- Implementar una red de comunicación segura y fiable entre todos los sistemas desarrollados.

\section{PROPUESTA}

Como se explicó anteriormente, el objetivo del proyecto es mejorar los procesos de gestión y de servicio al cliente de en un restaurante, para cumplir esta meta se propone la creación de una plataforma tecnológica que integre varios sistemas que suplan ciertas tareas del personal de servicio, optimizando así el tiempo y los recursos de trabajo. En la Fig. 1 se observa un esquema conceptual completo de la plataforma tecnológica propuesta. 


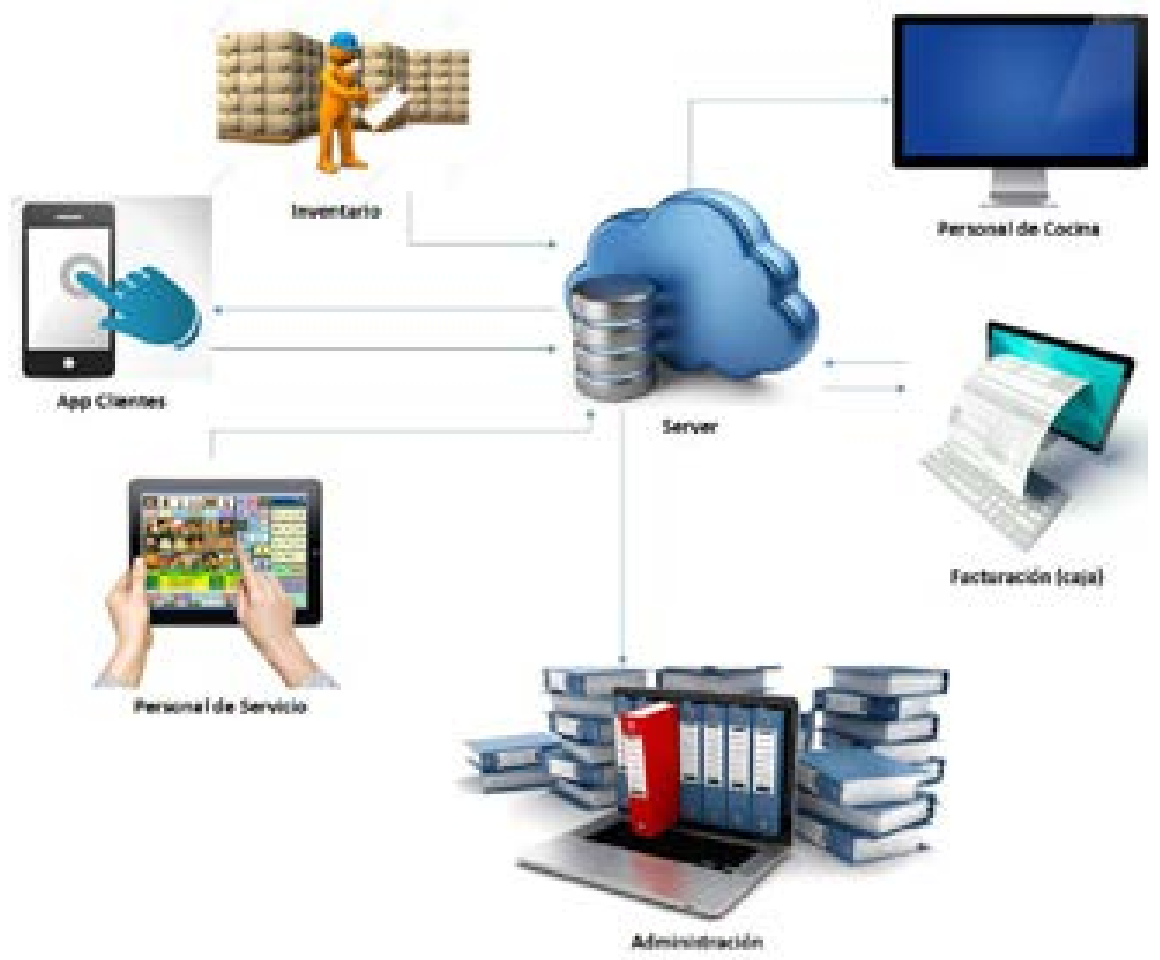

Figura 1. Esquema de bloques del sistema inteligente y colaborativo propuesto.

El servidor comandará las operaciones del sistema como peticiones y consultas y brindará la información de servicios a los distintos dispositivos COnectados a él; además, alojará la base de datos universal del sistema que será la encargada de almacenar toda la información relacionada con el funcionamiento del restaurante, la mínima información contenida deberá ser:
- Información de sucursales (direcciones, informaciones de contacto, nómina laboral, horarios, estado de funcionamiento).

- Inventario de productos (registro de productos en stock por cada sucursal, historial de ingreso y salida de productos).

- Base de datos de clientes (datos de facturación, registro de clientes con cuenta en la app móvil). 
- Dispositivos conectados (Registro de los dispositivos de servicio activos y en descanso, número de descargas de la app de clientes).

- Información por sucursal (pedidos realizados, pedidos en preparación, pedidos entregados).

- Información contable (registro de compras y ventas, historial de facturas emitidas, libro de contabilidad).

Los componentes del sistema propuestos son Is siguientes:

Administración.- El aplicativo de administración es el componente más importante después del servidor y la base de datos, se trata de un aplicativo web (en la Fig. 2 se ve un ejemplo para la portada del aplicativo) al que se pueda acceder desde cualquier dispositivo con conexión a internet, esto para facilitar el monitoreo y control por parte del dueño del establecimiento incluso estando a kilómetros de distancia del mismo. Las tareas que se pueden realizar desde este aplicativo serán:

- Visualización y administración (ingreso de productos) del inventario.

- Administración de la base de datos.

- Administración de productos (incluir o eliminar) y precios.

- Administración de procesos de servicio y de cocina (normas del restaurante).

- Monitoreo y control del registro de ventas.

- Monitoreo y control de recibos y facturas emitidas.

- Monitoreo y control de libros contables.

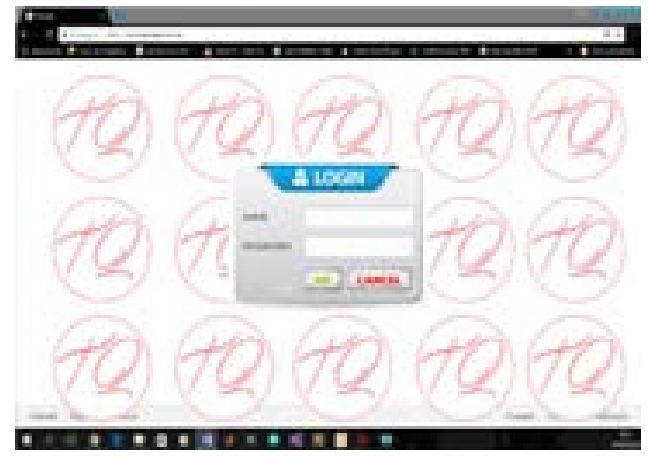

Fig. 2. Portada Aplicativo de Administración.

Facturación.- El sistema de facturación es el aplicativo que se utilizará en las cajas del restaurante, dependerá de la forma de compra del cliente; si se 
trata de un pedido para servir en el establecimiento la cuenta se enviará directamente del aplicativo del personal de servicio restando solo imprimir la factura; si el pedido se realiza para llevar o por llamada telefónica se debe generar la orden desde este aplicativo, por lo que la interfaz debe incluir las dos opciones para la generación de recibos de compra (ver Fig. 3).

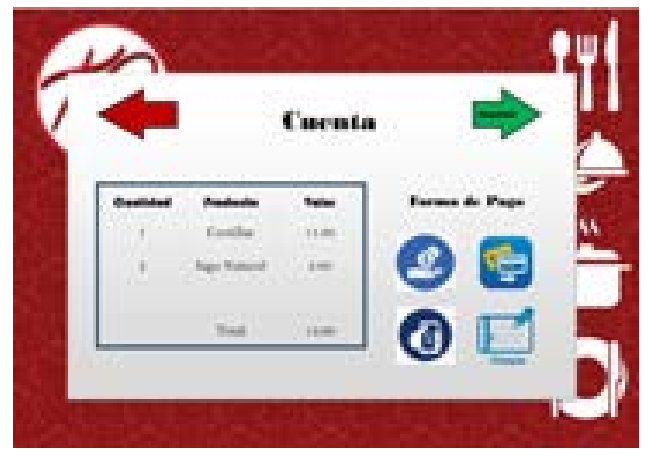

Figura 3. Propuesta de interfaz del servicio de facturación.

Personal de Servicio.- Se trata de un aplicativo Android que se instalará de preferencia en Tablets; incluirán todo el menú del restaurante para que los meseros puedan recoger los pedidos de los clientes (muy similar a la Fig. 3), los pedidos se registrarán según el número de mesa ocupada y la cuenta se enviará directamente al componente de facturación para la emisión del recibo de pago.
Personal de cocina.- Se trata de un sistema de visualización (Ver Fig. 4) en donde el personal de cocina pueda observar los pedidos realizados según el orden en que han sido generados, independientemente de si se han generado en mesa, para llevar o para domicilio. Cuando la orden esté preparada, el personal de cocina generará una alarma que alerte al personal de servicio para el paso de la comida y que elimine la orden del visualizador. 


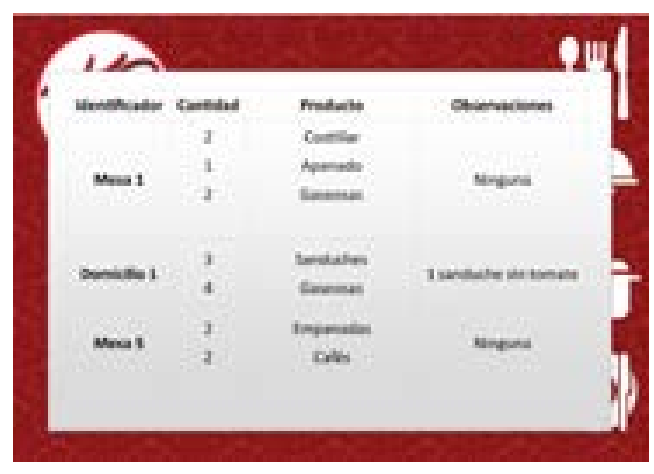

Fig. 4. Visualizador de cocina.

App clientes.- Es una aplicación móvil desarrollada para los dos sistemas operativos móviles más populares y que los clientes podrán descargar directamente de las tiendas oficiales, la aplica- ción cubre tareas como pedidos a domicilio, recepción de notificaciones con ofertas y promociones, generar cuentas de cliente regular, realizar comentarios y compartir experiencias en redes sociales.

\section{REQUERIMIENTOS}

Los requerimientos se levantan en forma de historias de usuario, estas deben contener las características específicas de los sistemas por implementar con el mayor detalle posible, las etapas sobre las cuales realizar el levantamiento de requerimientos son los siguientes:

- Servidor y base de datos.

- Sistema de administración.
- Sistema de facturación.

- Aplicativo de servicio.

- Cocina.

- Aplicativo clientes.

Lo más importante es identificar el mayor número de necesidades y desarrollar aplicativos que sean amigables con el usuario, es decir, no saturar las interfaces con funciones innecesarias. 


\section{INTERESADOS}

Identificar de manera adecua- interesados del proyecto definiendo vada los interesados del proyecto puede riables como la influencia o poder, el insignificar un gran avance en el éxito del terés y la estrategia de gestión para cada proyecto. En la Tabla 1 se identifica los grupo de interesados.

Tabla 1. Interesados o Stakeholders del proyecto.

\begin{tabular}{|c|c|c|c|c|}
\hline Nombre del Interesado & Rol & Poder & Interés & Estrategia \\
\hline Gerente Inventa S.T. & Patrocinador & Alto & Alto & Gestionar atentamente \\
\hline Delegado SENESCYT & Regulador & Bajo & Medio & Monitorear levemente \\
\hline Delegado financiero & Financista & Medio & Alto & Mantener Satisfecho \\
\hline Director de desarrollo & $\begin{array}{l}\text { Director del área de } \\
\text { desarrollo del sistema }\end{array}$ & Alto & Alto & Gestionar atentamente \\
\hline Director Administrativo & $\begin{array}{l}\text { Director del área admi- } \\
\text { nistrativa del proyecto }\end{array}$ & Alto & Alto & Gestionar atentamente \\
\hline Miembros del equipo técnico & $\begin{array}{l}\text { Desarrollo, dimensio- } \\
\text { namiento de equipos, } \\
\text { diseño gráfico. }\end{array}$ & Medio & Alto & Mantener Satisfecho \\
\hline Proveedores & Proveedor & Baja & Medio & Mantener Informado \\
\hline Personal de mercadeo & Vendedor & Baja & Alto & Mantener Satisfecho \\
\hline Propietarios de Restaurantes & Usuario Final & Bajo & Medio & Mantener Informado \\
\hline Administradores de Restaurantes & Usuario Final & Bajo & Bajo & Monitorear levemente \\
\hline $\begin{array}{l}\text { Personal de Servicio de un Res- } \\
\text { taurante }\end{array}$ & Usuario Final & Bajo & Bajo & Monitorear levemente \\
\hline
\end{tabular}




\section{GESTIÓN DE RRHH}

En la estructura organizacional de gestión del proyecto, se detalla cada órgano participativo, los roles, responsabilidades y perfiles requeridos para función dentro del proyecto. La figura de máxima autoridad de gestión del proyecto recae sobre el director de proyecto que a su vez deberá justificar sus decisiones sobre la base al consenso que se pueda lograr en el comité ejecutivo.

El comité ejecutivo está conformado por los líderes de cada paquete de trabajo y será presidido por el director de proyecto, sus funciones principales son:

- Analizar, evaluar y aceptar la propuesta de trabajo, incluyendo los objeti- vos, alcances y parámetros de éxito.

- Planificar las tareas y actividades para el desarrollo y monitoreo del proyecto.

- Aprobar los cronogramas de actividades y los recursos necesarios que se pretenden utilizar.

- Asegurarse de la marcha efectiva del proyecto.

- Revisar y evaluar los avances del proyecto.

- Solucionar los problemas que afecten el avance del proyecto.

En la Tabla 2 se describen los grupos de trabajo del proyecto y sus responsabilidades.

Tabla 2. Tabla de roles y funciones

\begin{tabular}{|c|c|}
\hline Grupos de Trabajo & Función \\
\hline Director del Proyecto & Encargado de gestionar el proyecto y asegurarse del cumplimiento del plan de proyecto. \\
\hline Director Administrativo y Financiero & $\begin{array}{l}\text { Encargado de las tareas administrativas y burocráticas del proyecto, genera informes de } \\
\qquad \text { estado y avance económico. }\end{array}$ \\
\hline Director Técnico & $\begin{array}{l}\text { Encargado de definir las técnicas, herramientas y el personal de desarrollo técnico, } \\
\text { asegurarse del nivel de calidad de los resultados. }\end{array}$ \\
\hline Líder de Paquete & $\begin{array}{l}\text { Coordinación de las tareas, interlocutor entre el equipo de trabajo y el director de } \\
\text { proyecto. }\end{array}$ \\
\hline Equipo de Trabajo & Ejecutores de las tareas de los distintos paquetes de trabajo, producen los entregables. \\
\hline
\end{tabular}


Como se puede observar en la Ta- para el proyecto. bla 3, se definieron 6 paquetes de trabajo

Tabla 3. Paquetes de Trabajo.

\begin{tabular}{|c|ccc}
\hline Identificador & Paquete de Trabajo & Inicio & Final \\
\hline PT1 & Gestión del Proyecto & M1 & M12 \\
\hline PT2 & Gestión de la Configuración & M1 & M12 \\
\hline PT3 & Análisis & M1 & M3 \\
\hline PT4 & Gesarrollo & M3 la Comunicación & M11 \\
\hline PT5 & Divulgación y Ventas & M1 & M12 \\
\hline PT6 & M12 \\
\hline
\end{tabular}

\section{GESTIÓN DE TIEMPO}

De acuerdo con las restricciones del proyecto, se estima una ejecución del mismo en un periodo de 12 meses, tomando como inicio el día 2 de octubre de 2017. Habiendo definido los paque- tes de trabajo en el subapartado anterior, ahora se puede definir un cronograma de actividades para el proyecto como el que se muestra en la Fig. 5.

\section{GESTIÓN DE COSTOS}

La elaboración del presupuesto incluye dos tipos de gastos: los directos y los indirectos. Los directos se desglosan en gastos de personal, materiales (consumibles), equipamiento (bienes amortizables), divulgación y otros gastos.
Para realizar un análisis de rentabilidad del proyecto se realiza una estimación de producción y ventas del sistema para cada período durante el tiempo de vida útil estimada del mismo; si se considera un tiempo de vida útil de 
10 semestres, cada período de análisis es 6 meses. Con los valores estimados se puede realizar el presupuesto previsivo para cada período, para esto se tomará en cuenta el valor unitario de cada sistema instalado.
Para analizar el beneficio esperado a lo largo de la vida de la plataforma tecnológica se realiza un análisis de beneficio, los resultados se muestran en la Tabla 4.

\section{Tabla 4. Beneficio y Rentabilidad}

\begin{tabular}{c|cccc} 
& Unidades Producidas & Unidades Vendidas & Beneficio & Rentabilidad (\%) \\
\hline Semestre 1 & 100 & 50 & 1811.84 & 2.6 \\
Semestre 2 & 200 & 150 & 14082.95 & 10.2 \\
Semestre 3 & 400 & 300 & 36317.65 & 13.2 \\
Semestre 4 & 500 & 350 & 49069.58 & 14.3 \\
Semestre 5 & 500 & 400 & 68450.00 & 20.0 \\
Semestre 6 & 400 & 500 & 85750.00 & 31.3 \\
Semestre 7 & 400 & 500 & 60529.41 & 22.1 \\
Semestre 8 & 300 & 500 & 46943.18 & 22.7 \\
Semestre 9 & 300 & 400 & 23947.83 & 11.6 \\
Semestre 10 & 200 & 200 & 7247.37 & 5.3
\end{tabular}

Se estima un beneficio de ciente para determinar el éxito en el ám3941 149. 81 dólares en el período de 5 bito económico, y en el sexto período se años. Por su parte, la rentabilidad del proyecto con esta estimación es muy baja al arranque del ejercicio comercial y es por la estimación en el beneficio que se ha definido para este período; en el cuarto espera que llegue a su máximo apogeo mercantil para empezar su declive en el período noveno. En la Fig. 6 se observa la gráfica de la evolución estimada de rentabilidad.

período se alcanza una rentabilidad sufi- 


\section{GESTIÓN DE CALIDAD Y RIESGOS}

Con el cumplimiento de los es- y al cierre del trabajo. Además este plan tándares y de las metodologías propues- brinda elementos de apoyo a la gestión tas se aspira obtener entregables de del proyecto.

mayor calidad a lo largo del ciclo de vida

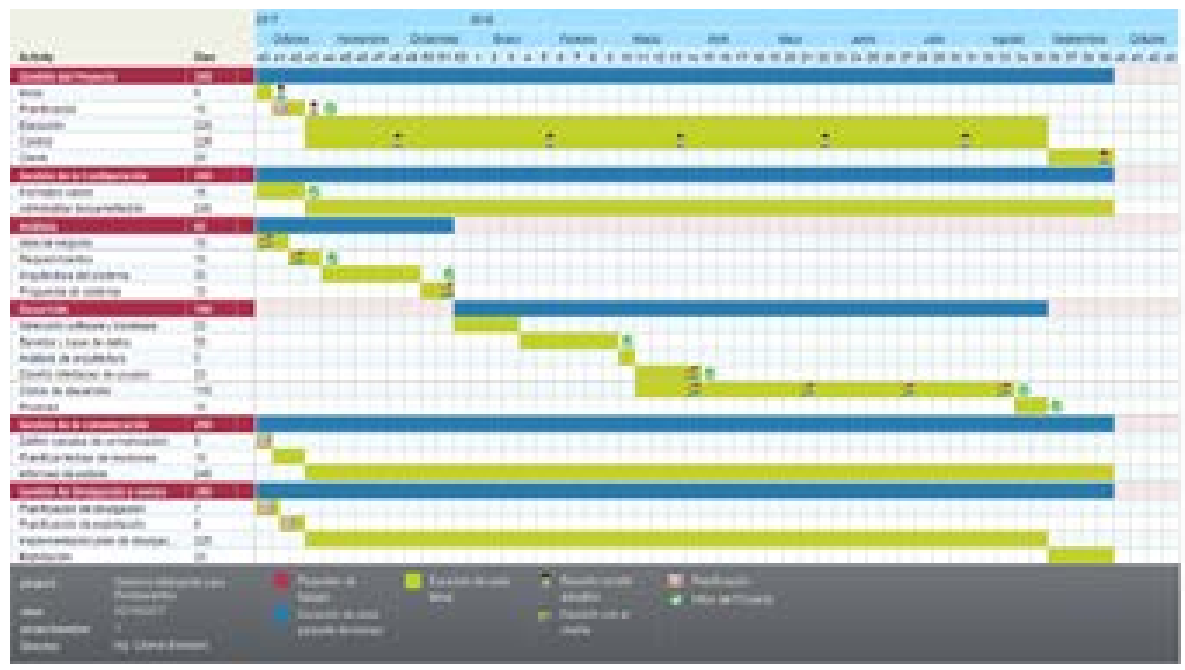

Figura 5. Cronograma de actividades.

La gestión de riesgos busca generar una cultura empresarial en la que la mayoría de los riesgos sean controlados y mitigados a fin de que la marcha del proyecto se vea lo menos impactada posible por este tema.

La identificación de riesgos se realizó considerando las actividades de mayor fallo en proyectos similares y según la experiencia de expertos en el tema de desarrollo informático. Los riesgos se identificaron en orden a partir de los paquetes de trabajo y de las tareas que compone cada uno. 


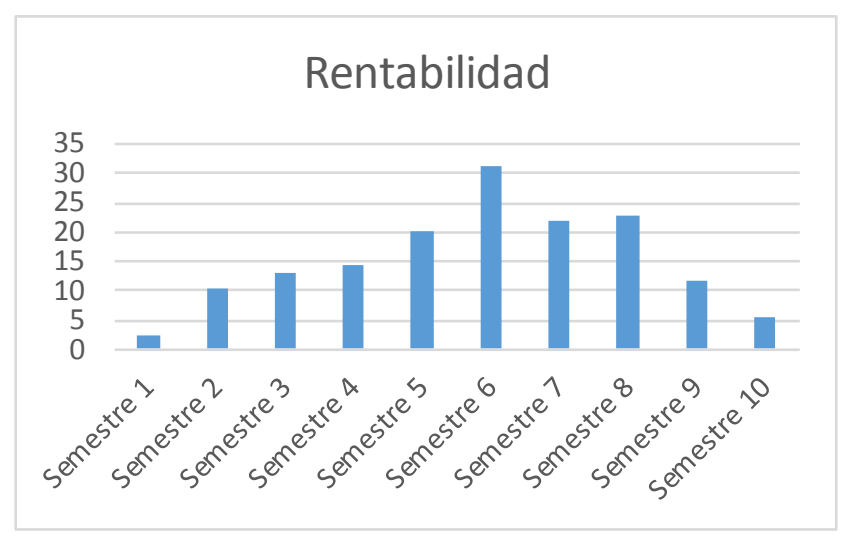

Fig. 6. Evolución prevista de la rentabilidad (\%)

\section{CONCLUSIONES}

Después del trabajo realizado, se ha podido generar un plan de proyecto que brinde las herramientas necesarias de gestión y control para la elaboración de un sistema inteligente que dé soporte a restaurantes en sus tareas de servicio y administración. Se desarrolló una propuesta inicial viable que, si bien podría cambiar en ciertos requerimientos en la etapa de desarrollo, representa una base sólida sobre la cual trabajar en la ejecución del proyecto.

Como conclusiones específicas del TFM se pueden comentar las siguientes: el aplicar metodologías ágiles brinda gran libertad de acción ante el rápido cambio de la tecnología en nuestros días, en especial la metodología scrum genera grandes ventajas al momento de desarrollar proyectos tecnológicos, como la detección temprana de dificultades en el desarrollo, rápida detección de metas imposibles, asignación de tareas a expertos para realización eficaz y eficiente y una autoorganización en donde los problemas de conflictos de autoridad quedan eliminados.

La combinación de metodologías puede ser muy beneficiosa en ciertos proyectos; en este trabajo se ha utilizado una metodología híbrida entre una metodología predictiva y scrum para planificar y gestionar el proyecto respectivamente, así se mantiene una planifica- 
ción general del proyecto, se fijan límites de calidad, tiempo y costes y posteriormente se da cierta libertad al equipo de trabajo al eliminar tareas administrativas innecesarias.

El levantamiento de requerimientos es una tarea fundamental en la planificación de proyectos tecnológicos. La descomposición del proyecto en fases (paquetes de trabajo) brinda mayor versatilidad y eficiencia en la gestión de cada una de las actividades que componen la ejecución del proyecto.
Se identificó a los involucrados del proyecto y se estimó sus aspiraciones para el presente proyecto. El identificar de manera temprana a los involucrados del proyecto (stakeholders) ayuda a tener una visión de las expectativas que se pueden generar y gestionarlas.

El desarrollo del cronograma de actividades es fundamental para el resto de la planificación del proyecto y para el éxito del mismo; se elaboró el presupuesto general del proyecto, así como un análisis de rentabilidad y beneficio esperado para los próximos 5 años. 


\section{BIBLIOGRAFÎA}

[1] INEN. (2015). Norma Técnica Ecuatoriana, Restaurantes, Sistema de gestión de la calidad y el ambiente. Quito, Ecuador.

[2] ARQHYS. (2012). Historia de los Restaurantes. México: Revista ARQHYS.com.

[3] Peter, P. J. $\neg$ Olson, J.C. (1990). Consumer Behaviour and Marketing Strategy. Homewood II.

[4] Anderson, E. W., Fornell, C., \& Rust, R. T. (1997). Customer Satisfaction, Productivity, and Profitability: Differences between Goods and Marketing Science.

[5] Molina, M. A. (2014). Satisfacción del Cliente y Rentabilidad. Victoria - Argentina: Universidad de San Andrés.

[6] Lledo, P. (2013). Director de Proyectos: Como aprovar el examnen PMP sin morir en el intento. Canada.

[7] Medina Martínez, Luisa Fernanada. (2016). Desarrollo de una Plataforma Tecnológica Colaborativa que promueva el uso de datos abiertos en Colombia. Bogotá.

[8] UNIR. (2016). Diseño, Planificación y Negociación de Presupuestos y Recursos. La Rioja.

[9] Crea Bussines Idea. (Julio 2009). Plan de Comunicación y Difusión. Madrid.

[10] PMI. (2013). Guía de los Fundamentos para la dirección de proyectos (PMBOK). FSC.

[11] Rios Pedromo, Karold Andrea. (2016). Plataforma tecnológica en gestión de proyectos para el parque científico de inovación social. La rioja: UNIR.

[12] Gallego, manuel Trigas. (2012). Gestión de Proyectos Informáticos.

[13] Ken Schwaber, Jeff Sutherland. (2016). La gúia definitiva de Scrum: Las reglas del juego.

[14] Tinjaca Peña, Diana Paola. (2016). Sistema Inteligente colaborativo para la optimización del uso del transporte público SICOUT. UNIR.

[15] Estrada-Hernández, J. A., \& León-Robaina, R. (2013). La integración de las tecnologías de información y comunicación en la gestión empresarial. Santiago.

[16] Manager, G. (2016). Gestión de proyectos con Scrum Manager. http://www. scrummanager.net/files/sm_proyecto.pdf.

[17] PPMC Consultores Internacionales Ltda. (2016). Manual de Estudio PMP. http://ppmci.com/projectmanagement/386/manual-de-estudio-pmp. html.

[18] Kerzner, H. R. (2013). Project management: a systems approach to planning, scheduling, and controlling: John Wiley \& Sons.

[19] Monge, E. C. (2010). Las estrategias competitivas y su importancia en la buena gestión de las empresas. Revista de Ciencias Económicas, 28(1).

[20] Roberts, P. (2007). Guide to project management: Achieving lasting benefit 
through effective change. John Wiley \& Sons.

[21] Universidad Internacional de la Rioja. (2016). Gestión de la Calidad, Riesgos y Evaluación. La Rioja.

[22] Universidad Internacional de la Rioja. (2016). Metodologías de gestión y dirección de proyectos. La Rioja.

[23] Universidad Internacional de la Rioja. (2016). Diseño, Planificación y Negociación de presupuestos y Recursos. La Rioja. 
ill|lil36 